\title{
Influencia del contenido en álcalis de los cementos sobre la corrosión de armaduras gaivanizadas
}

\author{
C. ANIRADE, Dra. en Química Industrial \\ Instituto Eduardo Torroja de la Construcción y del Cemento. Madrid \\ A. MACIAS, Leda. en Ciencias Químicas \\ Instituto Etunc rdo Torroja de la Construcción y del Cemento. Madrid
}

\section{R E S U M E N}

Las armaduras galvanizadas han mostrado un comportamiento errático en los ensayos llevados a cabo hasta ahora, encontrándose en la literatura datos muy contradictorios.

Los autores han encontrado que las siguientes tres variables tienen una decisiva importancia: a) $\mathrm{pH}$ del mortero u hormigón, que depende básicamente de la cantidad de álcalis del cemento, b) tipo de estructura metalográfica del recubrimiento galvanizado, que depende principalmente del tipo de acero y su contenido en $\mathrm{Si}$ y $\mathrm{C}$, y c) la humedad contenida en los poros del hormigón.

En este trabajo se presentan resultados relacionados sólo con el punto a). Se ha podido comprobar que existe un valor de $\mathrm{pH}$ umbral o crítico por encima del cual el recubrimiento galvanizado se corroe severamente, debido a la formación de capas no-protectoras de productos de corrosión.

La evolución da la velocidad de corrosión se ha seguido mediante la Resistencia de Polarización, y las pérdidas de peso se han determinado también, gravimétricamente.

\section{N T R O D U C C I O N}

Los favorables resultados obtenidos hasta el momento en la mayoría de las estructuras construidas con armaduras galvanizadas en medios y ambientes marinos (1) (2) son una prueba positiva del futuro de este método de protección de las armaduras.

Sin embargo, aun en la actualidad algunos países europeos se muestran muy reticentes a introducir esta innovación basándose en los resultados adversos de algunos investigadores (3), lo que ha desatado una viva polémica en la que las diversas posiciones en lugar de acercarse, tienden a radicalizars a cada vez más.

" Este trabajo ha sido presentado en la 13" International Galvanizing Conference. Londres. Mayo 1982. 
En nuestro país, el Centro de Investigaciones Metalúrgicas y el Instituto Eduardo Torroja de la Construcción y del Cemento, comenzaron en 1975 un programa de investigación con el objeto de conocer el comportamiento del galvanizado en contacto con hormigón y con el deseo de intentar aportar alguna luz en la controversia existente (4).

En esta investigación se ha podido constatar, como ya se ha publicado en anteriores ocasiones. que los resultados de la corrosión del galvanizado en hormigón eran mucho más erráticos y dispersos. en especial cuando existen $\mathrm{Cl}^{-}$en el hormigón (5) (6), que los del acero sin proteger.

También se pudo comprobar (además de otros extremos que no se abordan en el presente trabajo) que existen tres factores que en el caso del acero sin proteger no tienen incidencia en su velocidad de corrosión, pero que, en el caso del galvanizado, adquieren el carácter de variables que es necesario conocer y fijar para eliminar la dispersión y conseguir unos resultados reproducibles. Estos factores son: el tipo de cemento, la estructura metalográfica del recubrimiento galvanizado y la humedad contenida en los poros del hormigón.

En el presente trabajo se comunica un resumen de los primeros resultados obtenidos en el estudio sistemático de la influencia del tipo de cemento en la cinética de corrosión del acero galvanizado, utilizando la Resistencia de Polarización como técnica de medida.

Tradicionalmente se ha afirmado que la fase líquida del hormigón tenía un $\mathrm{pH}=12,6$ en base a que estaba compuesta principalmente por $\mathrm{Ca}(\mathrm{OH})_{2}$. Recientemente, diversos autores (9), en especial Longuet y otros (7) (8) han puesto de manifiesto que este $\mathrm{pH}$ podía alcanzar valores por encima de 13 , debido a que, con el paso del tiempo, el $\mathrm{Ca}(\mathrm{OH})_{2}$ desaparece de la disolución o fase líquida, que termina estando constituida principalmente por $\mathrm{NaOH}$ y $\mathrm{KOH}$.

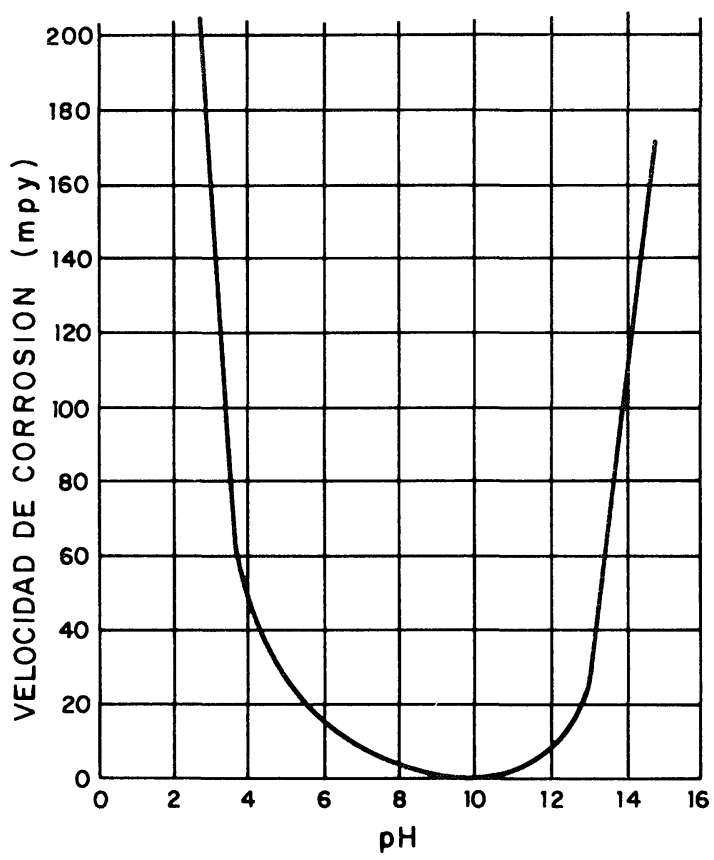

Fig. 1.-Velocidad de corrosión del $\mathrm{Zn}$ puro en función del $\mathrm{pH}$. de longitud. Tales aceros se galvanizaron por inmersión en caliente hasta obtener un recubrimiento de unas $60 \mu \mathrm{m}$ de espesor. El recubrimiento obtenido se muestra en la figura 2 y la composición química del acero se da en la tabla I. tudios de corrosión con acero desnudo, sin que hayan notificado o detectado que estas variaciones de $\mathrm{pH}$ modificaran sensiblemente la tradicional pasivación estable del acero. Sin embargo, en base a los estudios realizados por Roetheli, Cox y Littreal sobre Zn puro (figura 1), parecía lógico pensar que la velocidad de disolución del galvanizado debería elevarse notablemente con el aumento de sólo unas décimas de $\mathrm{pH}$.

Además del contenido en álcalis del cemento, son la proporción de este último y la relación a/c las que fijan el $\mathrm{pH}$ del hormigón.

\section{METODO EXPERIMENTAL}

\section{Materiales}

Se utilizaron aceros de dureza natural, corrugados, de $6 \mathrm{~mm}$ de diámetro nominal y $8 \mathrm{~cm}$

Los citados autores han realizado también es- 
TA B L A I

Composición química del acero

\begin{tabular}{|c|c|c|c|c|c|}
\hline$\% \mathbf{C}$ & $\% \mathrm{Si}$ & $\% \mathrm{Mn}$ & $\% \mathrm{P}$ & $\% \mathrm{~S}$ & $\% \mathrm{Cr}$ \\
\hline 0,25 & 0,39 & 1,30 & 0,014 & 0,028 & 0,08 \\
\hline
\end{tabular}

\section{Técnicas}

La velocidad de corrosión fue evaluada, como en anteriores trabajos (10), con un potenciostato AMEL con compensación electrónica de la caída ohmica-IR, mediante la determinación de la Resistencia de Polarización, $R_{\mathrm{p}}$, y por aplicación de la fórmula de Stern y Geary:

$$
I_{\mathrm{corr}}=\frac{\beta_{\mathrm{a}} \times \beta_{\mathrm{c}}}{2,3\left(\beta_{\mathrm{a}}+\beta_{\mathrm{c}}\right) R_{\mathrm{p}}}=\frac{B}{R_{\mathrm{p}}}
$$

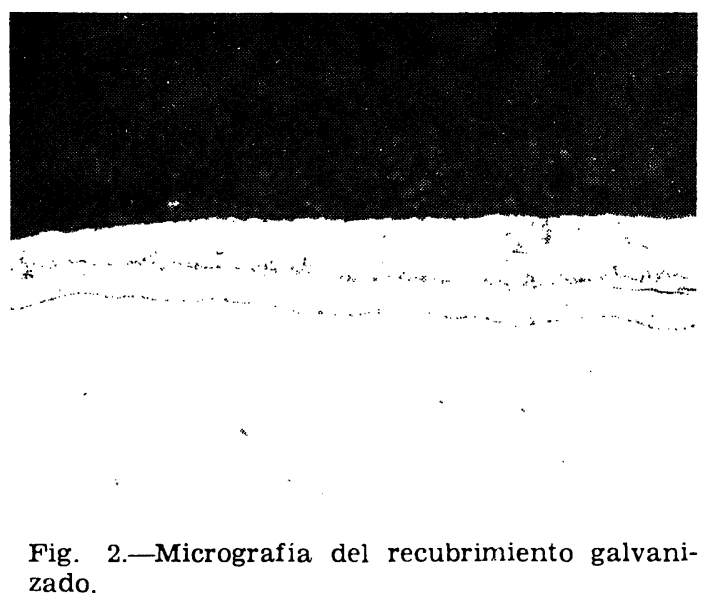

Las medidas de $\mathrm{pH}$ se realizaron con un electrodo combinado ORION de rango 0-14 y un Ionalizer Microprocesser ORION mod. 901.

El electrodo fue estandarizado con una disolución tampón de $\mathrm{pH}=13$.

Debido a la dificultad de medir directamente el $\mathrm{pH}$ en el mortero se prepararon suspensiones con los difenentes cementos de la siguiente manera: $25 \mathrm{~g}$ de cada cemento se agitaron con $50 \mathrm{ml}$ de agua destilada durante $15 \mathrm{~min}$. en un recipiente de polietileno cerrado. La suspensión se filtró inmediatamente bajo succión y en el filtrado se efectuó rápidamente (para evitar carbonatación) la medición de $\mathrm{pH}$.

\section{Tipos de probetas}

Se utilizaron dos tipos diferentes de probetas:

a) Células de corrosión de polietileno con 21 disoluciones diferentes todas saturadas en $\mathrm{Ca}(\mathrm{OH})_{2}$. La composición y $\mathrm{pH}$ de estas disoluciones se da en las tablas II y III. Las células se conservaron termostatadas a $25^{\circ} \mathrm{C}$ durante 33 días.

Este tipo de célula ha sido ya descrito con anterioridad (10) y se muestra en la figura 3.

b) Probetas de mortero, que al igual que en trabajos anteriores (10) fueron de $2 \times 5,5 \times$ $\times 8 \mathrm{~cm}$ con relación $\mathrm{a} / \mathrm{c}=0,5 \mathrm{y} \mathrm{c} / \mathrm{ar}=1 / 3$. Se fabricaron con nueve tipos diferentes de cemento, cuyo análisis químico y el $\mathrm{pH}$ de sus suspensiones se da en la tabla IV.

En cada probeta se embebieron dos redondos de acero y uno de grafito como electrodo auxiliar, tal y como muestra la figura 4.

Una serie de estas probetas se conservó durante 90 días al $100 \%$ de HR, y otra serie parcialmente sumergida en agua destilada en botes individuales de polietileno. 
TAB L A II

Composición, $\mathrm{pH}$ y comportamiento tipo de las disoluciones de $\mathrm{Ca}(\mathrm{OH})_{2}, \mathrm{NaOH}$ y $\mathrm{KOH}$

\begin{tabular}{|c|c|c|c|c|c|c|c|c|}
\hline \multirow[b]{2}{*}{\begin{tabular}{|l|} 
NUMERO \\
CELUL A \\
\end{tabular}} & \multicolumn{3}{|c|}{ CONCENTRACIONES AN̄ADIDAS } & \multirow[b]{2}{*}{$\begin{array}{l}\text { TIPO DE COM- } \\
\text { PORTAMIENTO } \\
\end{array}$} & \multirow[b]{2}{*}{$\mathrm{pH}$} & \multicolumn{3}{|c|}{ CONCENTRACIONES DETERMINADAS } \\
\hline & con. $\mathrm{Co}(\mathrm{OH})_{2}$ & $\begin{array}{c}\text { MOLARIDAD } \\
\mathrm{KOH}\end{array}$ & $\underset{\mathrm{NaOH}}{\text { MOLARIDAD }}$ & & & $\begin{array}{c}\text { MOL ARIDAD } \\
\mathrm{KOH}\end{array}$ & $\begin{array}{l}\text { MOLARIDAD } \\
\text { Na OH }\end{array}$ & $g / \mathrm{Ca}^{2+}$ \\
\hline 1 & sot. & 0,1 & - & A & 12,97 & & & 0,258 \\
\hline 2 & sot. & 0,5 & - & c & 13,59 & & & 0,053 \\
\hline 3 & sot. & 1 & - & c & 13,89 & 1,10 & & 0,032 \\
\hline 4 & sot. & 1,5 & - & c & 14,00 & 1,48 & & 0,022 \\
\hline 5 & sot. & - & - & A & 12,60 & & & 0,892 \\
\hline 6 & sot. & - & 0,01 & A & 12,61 & & & 0,722 \\
\hline 7 & sat. & - & 0,05 & A & 12,80 & & & 0,408 \\
\hline 8 & 804. & - & 0,1 & A & 12,99 & & & 0,220 \\
\hline 9 & sot. & - & 0,2 & B & 13,17 & & 0,25 & 0,112 \\
\hline 10 & $30 t$. & 0,5 & 0,05 & c & 13,61 & & & 0,048 \\
\hline 11 & $30 t$ & 0,7 & - & c & 13,72 & & & 0,049 \\
\hline 12 & sot. & 0,35 & - & c & 13,45 & & & 0,072 \\
\hline 13 & sot. & 0,2 & - & B & 13,24 & & & \\
\hline 14 & sot. & 0,6 & 0,1 & c & 13,69 & & & 0,038 \\
\hline 15 & sat. & - & 0,5 & c & 13,50 & & 0,5 & 0,050 \\
\hline
\end{tabular}

T A B L A III

Composición, $\mathrm{pH}$ y comportamiento tipo de las disoluciones con $\mathrm{Ca}(\mathrm{OH})_{2}, \mathrm{SO}_{4} \mathrm{Ca}, \mathrm{SO}_{4} \mathrm{Na}_{2}$ y $\mathrm{SO}_{4} \mathrm{~K}_{2}$

\begin{tabular}{|c|c|c|c|c|c|c|c|c|c|c|}
\hline \multirow[b]{2}{*}{$\begin{array}{l}\text { NUMERO } \\
\text { CELULAA }\end{array}$} & \multicolumn{4}{|c|}{ CONCENTRACIONES } & \multirow[b]{2}{*}{$\begin{array}{l}\text { TIPO DE COMA } \\
\text { PORTAMENTIO }\end{array}$} & \multirow[b]{2}{*}{ DH } & \multicolumn{2}{|c|}{ CONCENTRACIONES } & \multicolumn{2}{|c|}{ DETERMINADAS } \\
\hline & con. $\mathrm{Co}(\mathrm{OH})_{2}$ & $9 / \mathrm{I} \mathrm{SO}_{4} \mathrm{Co}$ & $0 / \mathrm{SO}_{4} \mathrm{~K}_{2}$ & $9 / \mathrm{SO}_{4} \mathrm{No}_{2}$ & & & $\mathrm{~g} \wedge \mathrm{Co}^{2+}$ & $g / 1 \mathrm{k}^{+}$ & $\mathrm{g} / \mathrm{L} \mathrm{No}{ }^{+}$ & $\mathrm{g} / \mathrm{L} \mathrm{SO}=$ \\
\hline 16 & 804. & 5 & - & - & A & 12,43 & 1,132 & - & - & 0,94 \\
\hline 17 & $80 t$. & - & - & 5,22 & A & 12,70 & 0,856 & - & 1,75 & 2,56 \\
\hline 18 & 801. & - & 6,41 & - & A & 12,77 & 0,784 & 3,13 & - & 2,48 \\
\hline 19 & sot. & 5 & - & 5,22 & $A / B$ & 12,71 & 0,858 & - & 1,84 & 2,41 \\
\hline 20 & sot. & 5 & 6,41 & - & $A / B$ & 12,77 & 0,872 & 2,37 & - & 2,51 \\
\hline 21 & 801. & 5 & 6,41 & 5,22 & B & 12,72 & 0,480 & 2,84 & 1,62 & 5 \\
\hline
\end{tabular}


T A в L A IV

Análisis químico de los nueve cementos ensayados y $\mathrm{pH}$ de sus suspensiones

\begin{tabular}{|c|c|c|c|c|c|c|c|c|c|}
\hline $\begin{array}{c}\text { CEMENTOS } \\
\%\end{array}$ & $\begin{array}{c}1 \\
P-350\end{array}$ & $\begin{array}{c}2 \\
P-450 \\
\end{array}$ & $\begin{array}{c}3 \\
(P-350-Y)_{1}\end{array}$ & $\begin{array}{c}4 \\
(P-450-Y)_{2}\end{array}$ & $\begin{array}{c}5 \\
\text { (PUZ-350) }\end{array}$ & $\begin{array}{c}6 \\
\text { (PUZ-35O) } \\
\end{array}$ & $\begin{array}{c}7 \\
(S-I)_{1}\end{array}$ & $\begin{array}{c}8 \\
(S-I)_{2}\end{array}$ & $\begin{array}{c}9 \\
P-450-A R I\end{array}$ \\
\hline P. F. & 2,20 & 3,60 & 3,03 & 0,60 & 2,74 & 2,60 & - & - & 2,90 \\
\hline R. 1 . & 0,15 & 1,20 & 2,74 & 0,40 & 1,89 & 5,87 & 1,17 & 2,99 & 1,40 \\
\hline $\mathrm{SiO}_{2}$ & 20,00 & 18,50 & 20,40 & 20,10 & 21,28 & 25,77 & 19,77 & 19,95 & 18,60 \\
\hline $\mathrm{Al}_{2} \mathrm{O}_{3}$ & 6,02 & 4,90 & 4,53 & 4,30 & 5,05 & 6,91 & 7,54 & 4,67 & 4,8 \\
\hline $\mathrm{Fe}_{2} \mathrm{O}_{3}$ & 3,21 & 3,00 & 5,85 & 6,60 & 11,14 & 3,75 & 15,88 & 11,78 & 4,00 \\
\hline $\mathrm{CoO}$ & 64,00 & 63,30 & 58,93 & 64,30 & 52,39 & 46,96 & 48,14 & 52,24 & 62,30 \\
\hline $\mathrm{MgO}$ & 1,80 & 1,20 & 1,40 & 1,10 & 1,88 & 3,38 & 1,30 & 2,06 & 1,2 \\
\hline $\mathrm{SO}_{3}$ & 2,86 & 3,60 & 2,67 & 2,40 & 2,70 & 2,95 & 2,31 & 2,56 & 3,50 \\
\hline O Ca LIBRE & 1,72 & 0,90 & 0,98 & 0,50 & 2,31 & 1,62 & 0,73 & 2,56 & 1,10 \\
\hline $\mathrm{Na}_{2} \mathrm{O}$ & 0,18 & 0,14 & 0,11 & 0,10 & 0,15 & 0,48 & 0,24 & 0,14 & 0,23 \\
\hline$K_{2} O$ & 0,39 & 0,78 & 0,30 & 0,68 & 0,37 & 0,84 & 0,63 & 0,36 & 1,00 \\
\hline MnO & - & - & - & - & - & - & - & 0,029 & - \\
\hline$s \pm$ & - & - & - & - & - & - & 0,75 & 0,23 & - \\
\hline $\mathrm{CrO}_{3}(p p a)$ & 8 & 12 & 8 & 8 & 8 & 8 & 8 & 8 & 20 \\
\hline $\begin{array}{l}\text { PH DE SUS- } \\
\text { PENSIONES }\end{array}$ & 12,30 & 12,37 & 12,11 & 12,40 & 12,23 & 12,44 & 12,15 & 12,19 & 12,62 \\
\hline
\end{tabular}

\section{RESULTADOS}

\section{Ensayos en disolución}

En la figura 5 se muestran los $\mathrm{pH}$ que se producen al añadir diversas concentraciones de $\mathrm{NaOH}, \mathrm{KOH}$ y $\mathrm{SO}_{4}{ }^{=}$a la disolución saturada de $\mathrm{Ca}(\mathrm{OH})_{2}$.

En la figura 6 se han representado los valores de $i_{\text {corr }}$ y del $E_{\text {corr }}$ a la 1 hora de inmersión de los redondos en las 21 disoluciones ensayadas (los números se refieren a los de las disoluciones de las tablas II y III). Cada valor es la media de tres redondos. Al aumentar el valor del $\mathrm{pH}$, el $E_{\text {corr }}$ se desplaza hacia valores más negativos y la $i_{\text {corr }}$ llega a multiplicarse hasta por un factor de 10 . El cambio brusco con el $\mathrm{pH}$ parece producirse a partir de 13,3. Los valores de $i_{\text {corr }}$ obtenidos son ligeramente inferiores a los de Roetheli, Cox y Littreal (11) de la figura 1. 


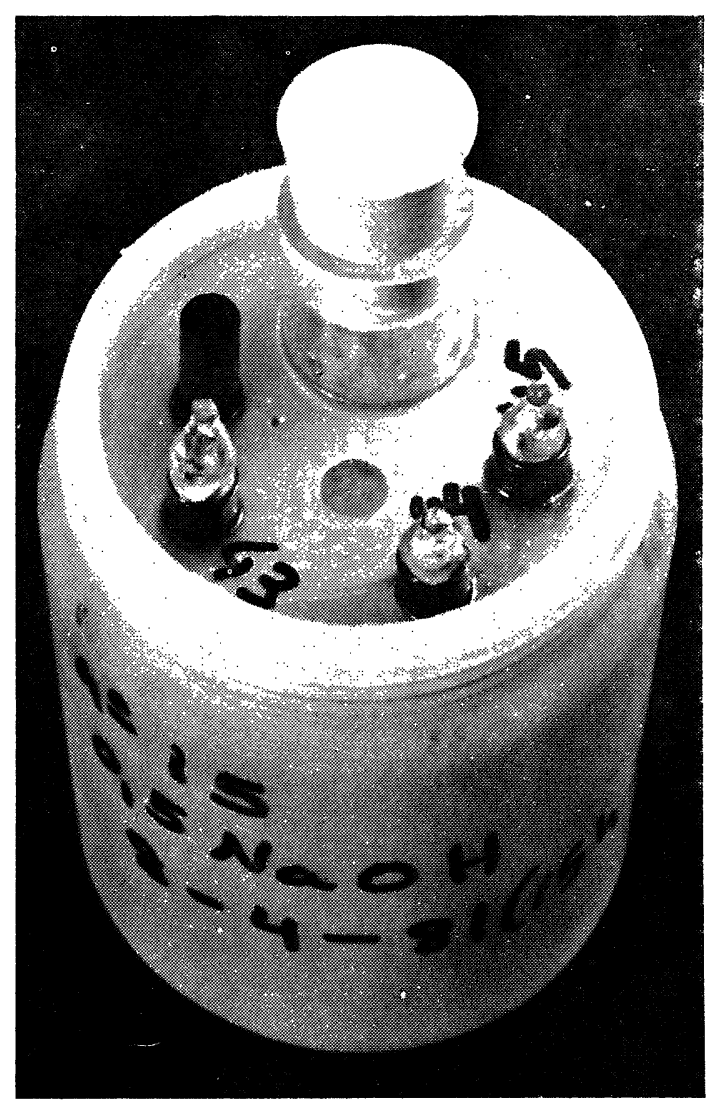

Fig. 3.-Célula de corrosión.

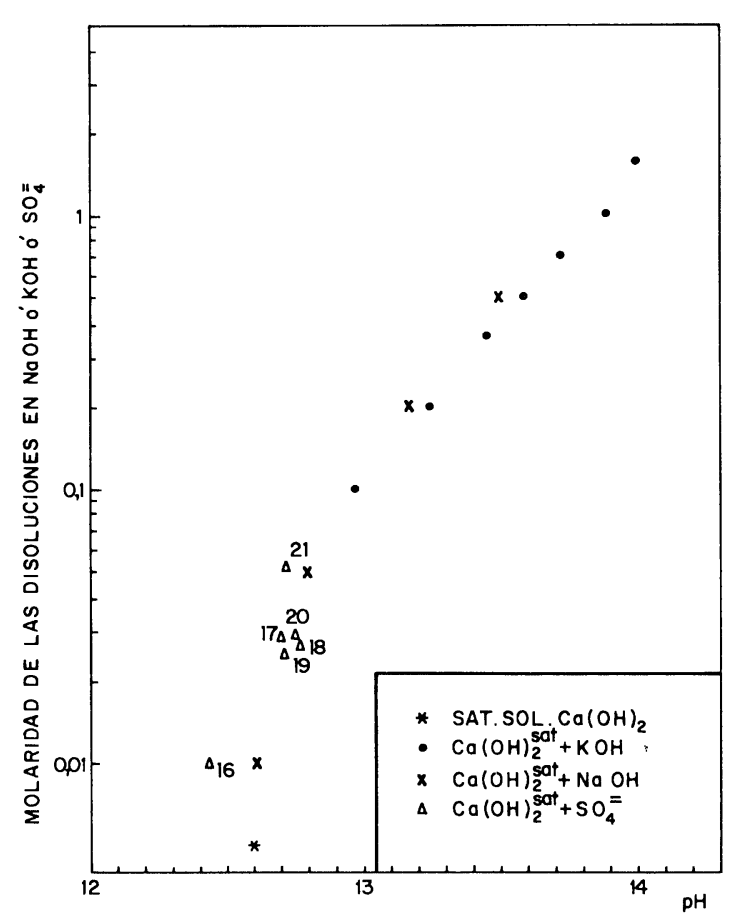

Fig. 5.- $\mathrm{pH}$ de las 21 disoluciones ensayadas en función de la adición de $\mathrm{NaOH}$, $\mathrm{KOH}$ y $\mathrm{SO}_{4}$ " a la disolución saturada de la $\mathrm{Ca}(\mathrm{OH})_{2}$.

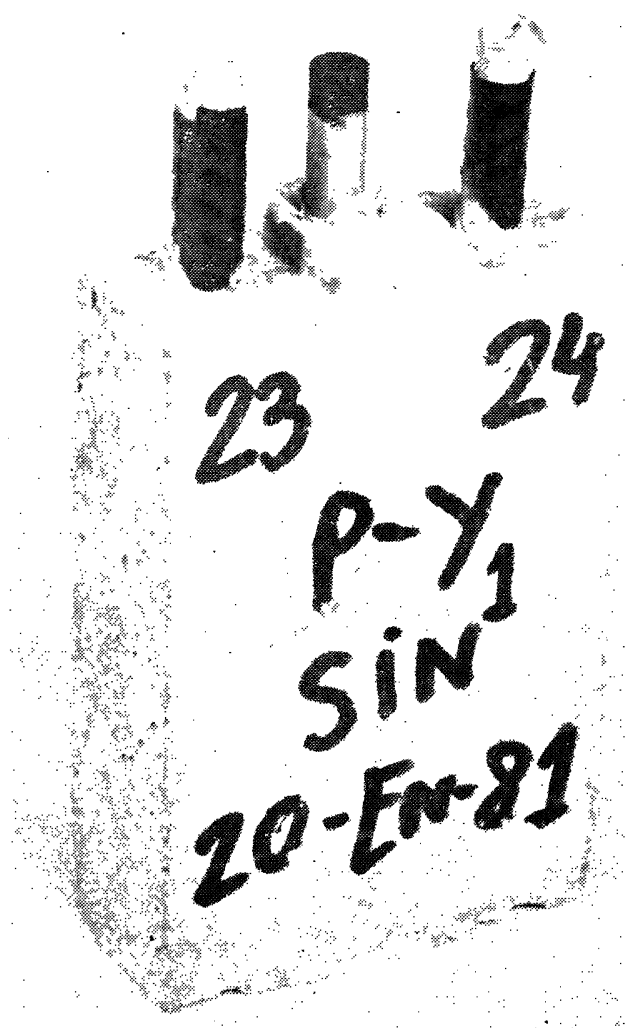

Fig. 4.-Probeta de mortero.

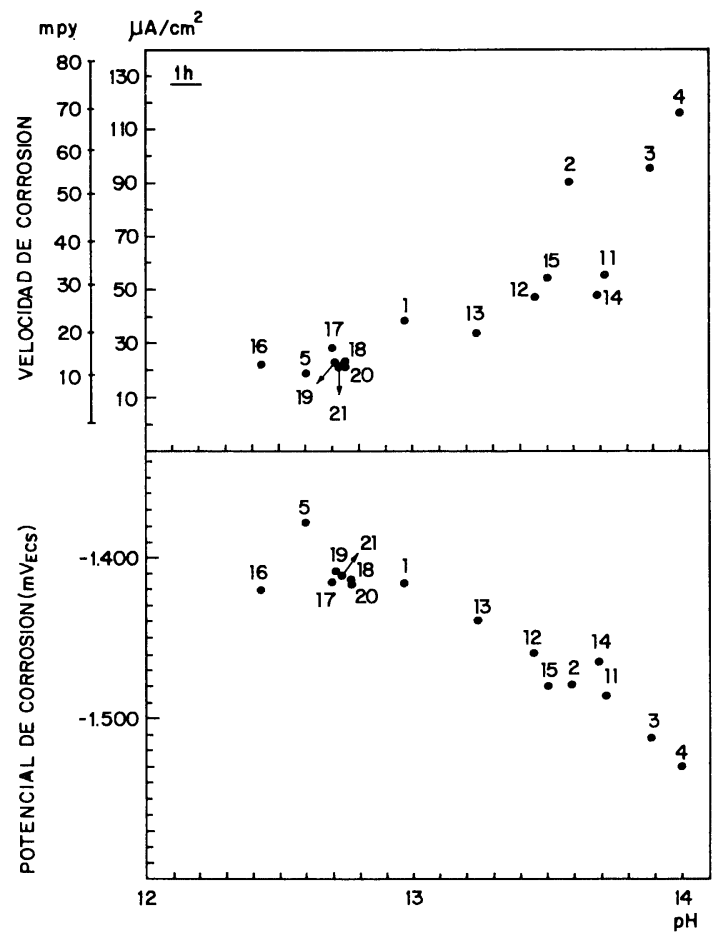

Fig. 6.-Valores de $i_{\text {corr }}$ y $E_{\text {corr }}$ después de $1 \mathrm{~h}$ de inmersión en función del pH de las disoluciones (los números corresponden a los indicados en las tablas II y III).

Materiales de Construccion N. 184 - 1981 
En la figura 7 se han resumido los tres comportamientos básicos frente al tiempo de los redondos galvanizados durante los 33 días del ensayo: en todos los casos se produce una fuerte corrosión inicial acompañada de un desprendimiento de $\mathrm{H}_{2}$. Posteriormente, unos redondos alcanzan con más (comportamiento $A$ ) o menos (comportamiento $B$ ) rapidez un estado de aparente pasividad, mientras que otros (comportamiento $C$ ) a los 33 días se siguen corroyendo a velocidades notables. Se han incluido en las tablas II y III los comportamientos ( $A, B$ ó $C$ ) obtenidos con cada disolución.

Los valores de $i_{\text {, .... y }} E_{\text {, nr r }}$ después de 33 días de inmersión se dan en la figura 8 . El valor de $\mathrm{pH}=13,3$ define dos zonas: una de formación de películas protectoras de productos de corrosión a $\mathrm{pH}<13,3$ con $E_{\text {corr }}=-450 \ldots-650 \mathrm{mV}_{\mathrm{ECS}}$ e $i_{\text {corr }}=0,1 \ldots 0,8$ $\mu \mathrm{A} / \mathrm{cm}^{2}$, y otra de formación de productos de corrosión no protectores a $\mathrm{pH}>13,3$ con $E_{\text {corr }}=-925 \ldots-1.120 \mathrm{mV}_{\mathrm{FCS}}$ e $i_{\text {corr }}=5 \ldots 15 \mu \mathrm{A} / \mathrm{cm}^{2}$.

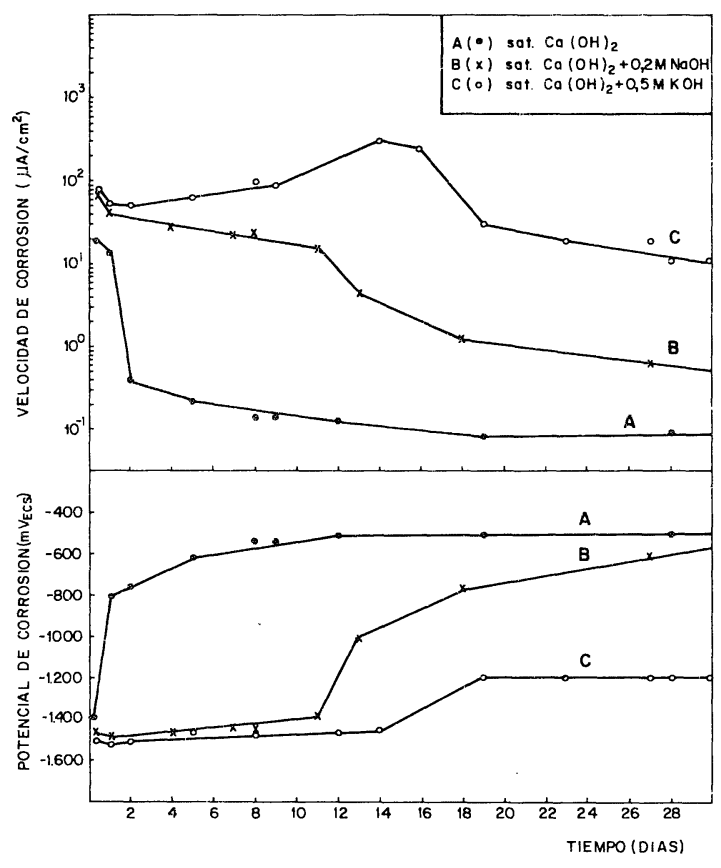

Fig. 7.-Comportamiento tipo de la evolución de la $i_{\text {curr }}$ y $E_{\text {corr }}$ con el tiempo de los redondos galvanizados en las 21 disoluciones.

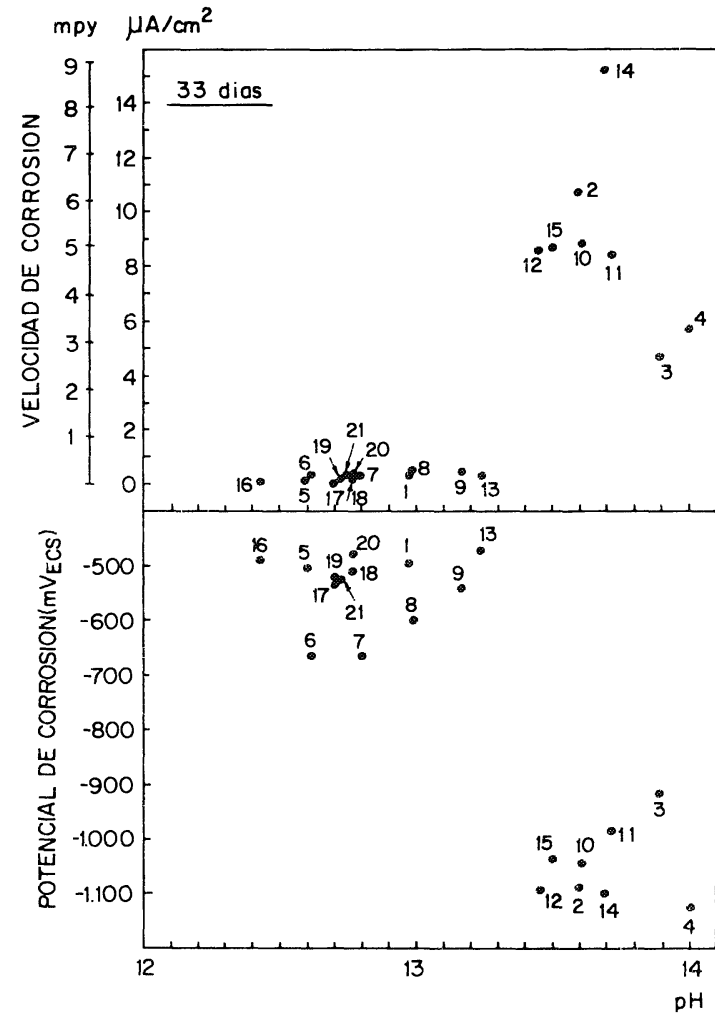

Fig. 8.-Valores de $i_{\text {corr }}$ y $E_{\text {corr }}$ a los 33 días del ensayo en función del $\mathrm{pH}$ de las disoluciones.

Los difractogramas de $\mathrm{RX}$ de los productos de corrosión de los redondos sumergidos en la disolución saturada de $\mathrm{Ca}(\mathrm{OH})_{2}$, muestran la presencia de un producto similar al identificado como hidroxicincato cálcico por Lieber y Gebauer (12) y Rehm y Lämmke (13), figura 9.

Los productos de corrosión formados en las disoluciones con adiciones de $\mathrm{NaOH}, \mathrm{KOH}$ y $\mathrm{SO}_{4}{ }^{\prime}$ parecen morfológicamente diferentes a los de la figura 9.

\section{Ensayo en mortero}

En la figura 10 se ha representado el $\mathrm{pH}$ de las suspensiones de los cementos frente al contenido en $\mathrm{K}_{2} \mathrm{O}$ (el componente alcalino mayoritario en los cementos españoles) y de ál- 
calis totales (expresado como $\mathrm{Na}_{2} \mathrm{O}+0,658 \mathrm{~K}_{2} \mathrm{O}$ ). Es clara la tendencia a un aumento del $\mathrm{pH}$ con el aumento en el contenido en álcalis totales de los cementos.

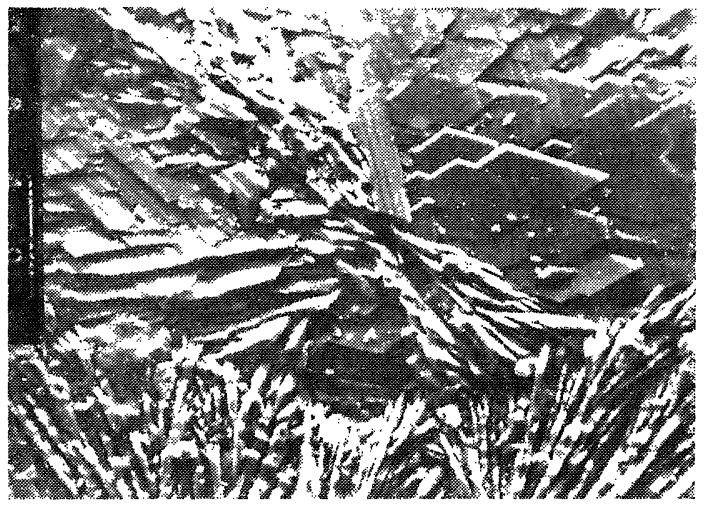

Fig. 9.-Producto de corrosión obtenido en la disolución saturada de $\mathrm{Ca}(\mathrm{OH})_{2}$, que es morfológica y espectralmente similar al hidróxicincato cálcico.

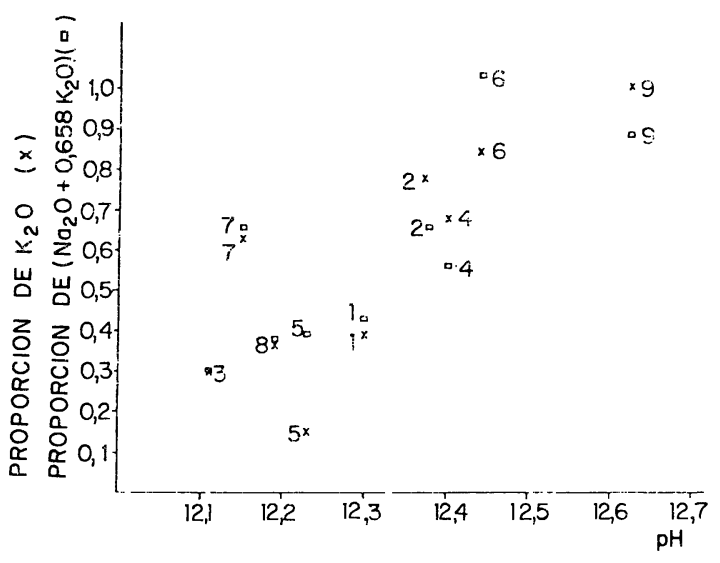

Fig. 10. $-\mathrm{pH}$ de las suspensiones de cemento en función del contenido en $\mathrm{K}_{2} \mathrm{O}$ o en álcalis totales expresado como $\left(\mathrm{Na}_{2} \mathrm{O}+0,658 \mathrm{~K}_{2} \mathrm{O}\right)$.

TA B L A V

Comparación entre el espaciado obtenido por Rehm y Lämmke (13) para el hidroxicincato cálcico $y$ el obtenido en el presente ensayo

\begin{tabular}{|l|l|l|l|l|l|l|l|l|l|}
\cline { 2 - 9 } \multicolumn{1}{c|}{} & 14,2 & 17,7 & 21,5 & 22,6 & 28,5 & 30,9 & 36,6 & 38,9 & 46,6 \\
\hline $\begin{array}{c}d \\
\text { nuestra muestra }\end{array}$ & 6,22 & 5,00 & 4,11 & 3,91 & 3,11 & 2,88 & 2,459 & 2,3166 & 1,9473 \\
\hline $\begin{array}{c}d \\
\text { Rehm-Lämmke (13) }\end{array}$ & 6,2369 & 5,0107 & 4,1329 & 3,9342 & 3,1318 & 2,8938 & 2,4551 & 2,3151 & 1,9489 \\
\hline
\end{tabular}

La evolución a lo largo del tiempo de la $i_{c o r r}$ y del $E_{c a r r}$ de los redondos galvanizados embebidos en el mortero fabricado con el cemento P-450, se ha representado en la figura 11. La evolución de ambas variables es similar para todos los cementos ensayados, si bien los valores que adquiere la $i_{c o r r}$ son diferentes, tal y como muestra la figura 12 donde se ha representado el valor de la $\dot{i}_{\text {corr }}$ a $\operatorname{los} 1,28$ y 90 días frente al $\mathrm{pH}$ de las suspensiones de los cementos (los números corresponden a los cementos de la tabla IV).

Puede deducirse de esta figura que a las $24 \mathrm{~h}$ de amasado parece existir un valor de $\mathrm{pH}$ a partir del cual crece mucho la $i_{\text {corr }}$. A los 28 días la situación es similar a la de los 90 días, comprobándose una paulatina elevación de la $i_{c o r r}$ con el aumento de $\mathrm{pH}$ aunque sin ningún "umbral" definido. Los valores de $i_{r, r r}$ mostrados por todos los cementos a los 90 días indican una velocidad de corrosión ya muy pequeña.

El ataque total sufrido por las armaduras durante los 90 días del ensayo puede apreciarse en la figura 13 donde se ha representado la pérdida gravimétrica en $\mathrm{mg} / \mathrm{cm}^{2}, \mathrm{mpy}$ y en $\mu \mathrm{m} / \mathrm{año}$ frente a los $\mathrm{pH}$ de las suspensiones de los cementos. Destaca el mayor ataque sufrido por los cementos 7 y 8 (ambos de escorias de alto horno), respecto de lo que les correspondería por el $\mathrm{pH}$ que exhiben sus suspensiones y que posiblemente sea debido a la presencia de los $\mathrm{S}^{-}$de las escorias. 


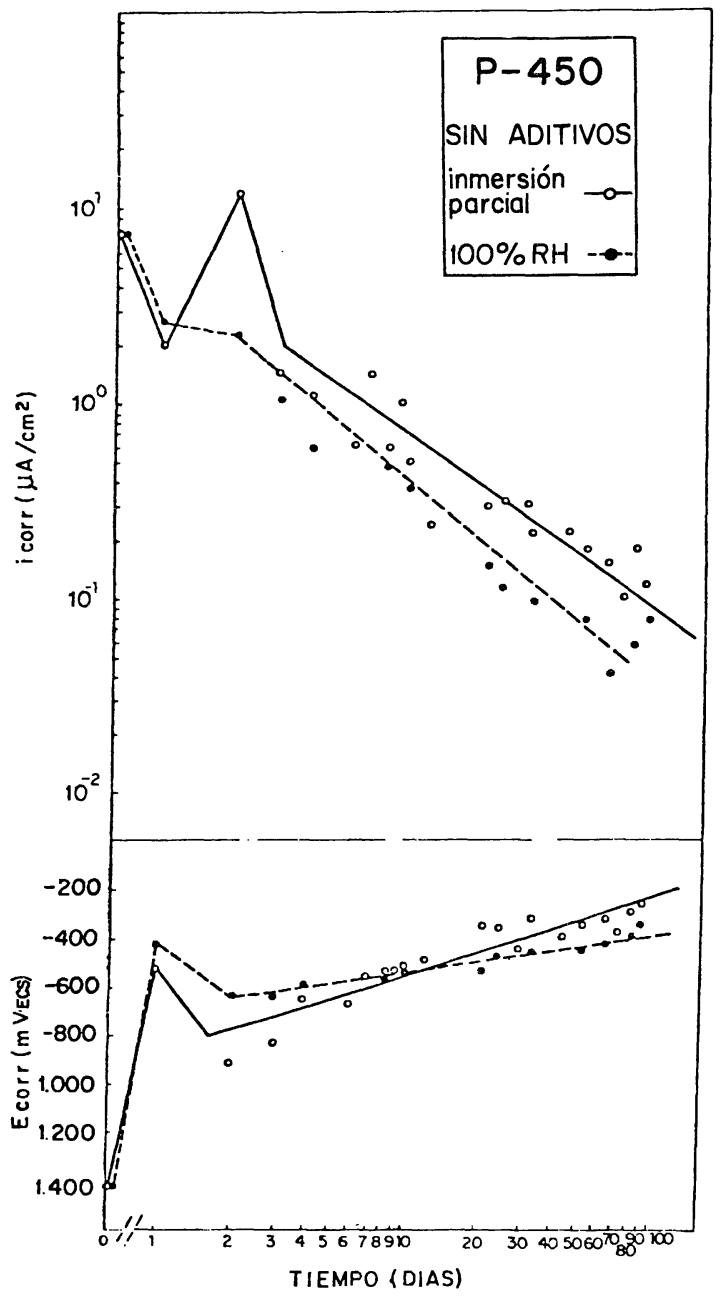

Fig. 11.-Evolución de $i_{\text {corr }}$ y $E_{\text {corr }}$ con el tiempo de los redondos galvanizados embebidos en mortero fabricado con cemento P-450.

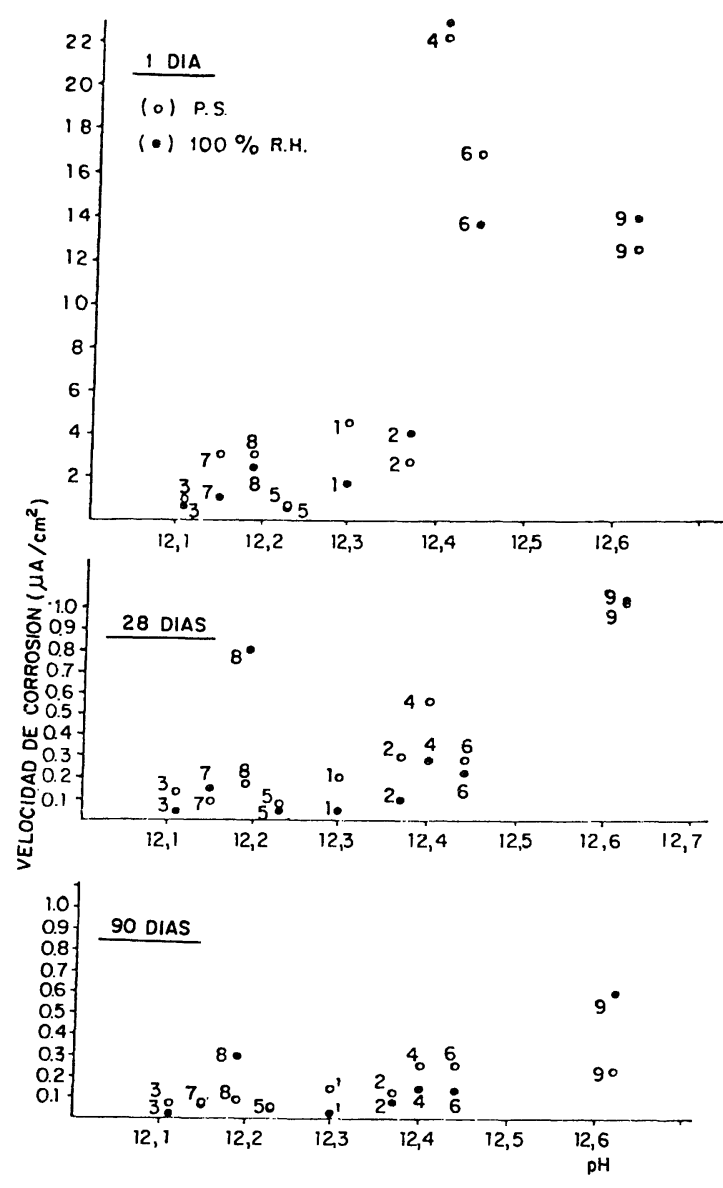

Fig. 12.-Valores de la $i_{\text {corr }}$ y el $E_{\text {corr }}$ a las $24 \mathrm{~h}$, 28 dias y 90 días en función del pH de las suspensiones de los cementos (los números corresponden a los de la taba IV).

\section{Comparación entre los resultados gravimétricos y electroquímicos}

En la figura 14 se muestra la comparación entre las pérdidas gravimétricas y las calculadas a partir de los datos de $\mathrm{Rp}$ de los ensayos en disolución. En el presente caso, los mejores resultados se obtienen con $B=13$ $\mathrm{mV}$ para el estado activo y $B=52 \mathrm{mV}$ para el pasivo.

En la figura 15 se realiza la misma comparación para los ensayos en mortero. En este caso los mejores resultados se obtienen con un valor de $B=26 \mathrm{mV}$ para el estado activo $\left(i_{\text {corr }}>0,5 \mu \mathrm{A} / \mathrm{cm}^{2}\right)$ y $B=52 \mathrm{mV}$ para el pasivo. La concordancia obtenida en los ensayos en mortero es peor que en los ensayos en disolución, posiblemente debido al error que se comete, a pesar de la eliminación instrumental de la caída óhmica RI, por la elevada resistividad del mortero.

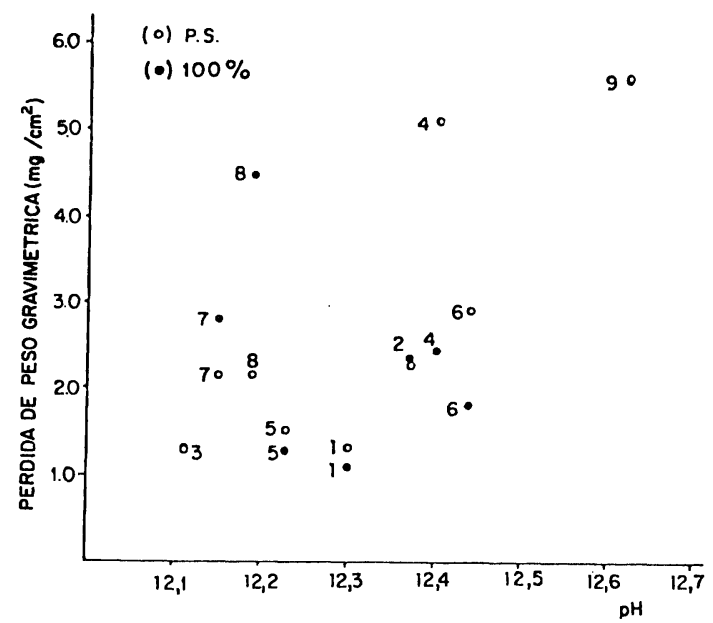

Fig. 13.-Pérdida gravimétrica en función de los pH de las suspensiones de los cementos. 


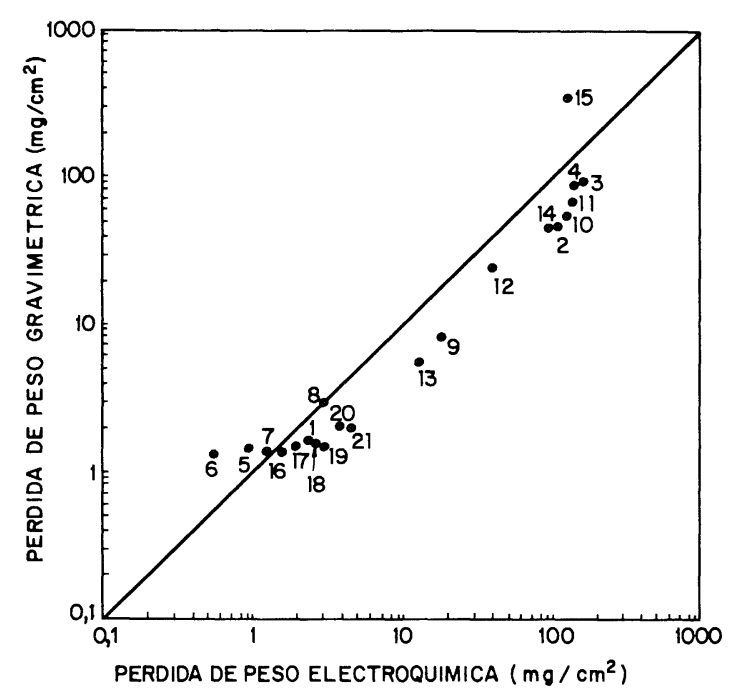

Fig. 14.-Comparación entre las pérdidas gravimétricas y las calculadas por vía electroquímica de los ensayos realizados en disolución.

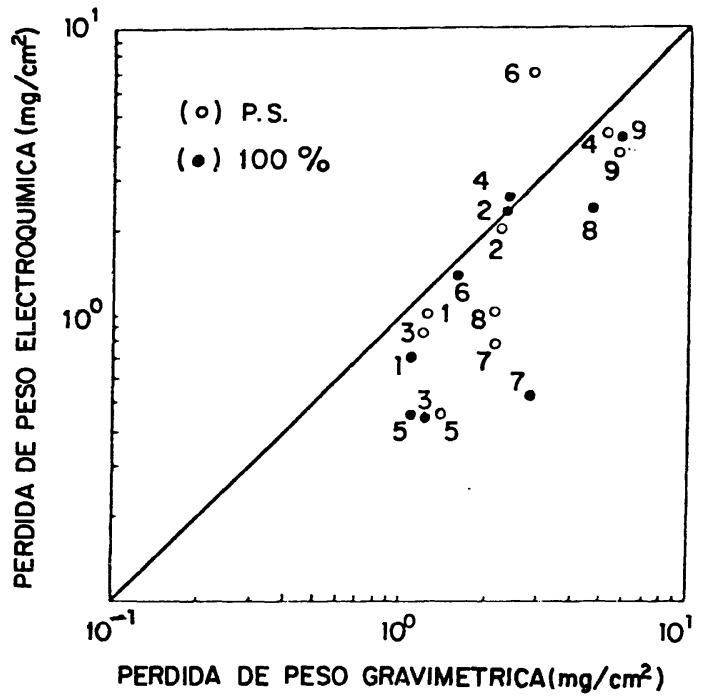

Fig. 15.-Comparación entre las pérdidas gravimétricas y las calculadas por vía electroquímica de los ensayos en mortero.

También es de destacar la muy aceptable concondancia entre los resultados de la figura 6, deducidos aquí por vía electroquímica y los de la figura 1 determinados por sus autores con metodología diferente.

\section{DISCUSION}

En el presente trabajo se ha podido establecer en los ensayos en disolución, que existe un pH umbral, a partir del cual con un aumento de pocas décimas de $\mathrm{pH}$, la $i_{\text {corr }}$ del acero galvanizado aumenta drásticamente.

Los ensayos en mortero, no han resultado en cambio tan concluyentes e ilustrativos como los obtenidos en disolución, debido a la limitación de no poder determinar el $\mathrm{pH}$ del mortero directamente y tener que recurrir a estimarlo a partir del pH de una suspensión. Es conocido que como el $\mathrm{pH}$ es una magnitud que varía con la dilución, los $\mathrm{pH}$ de las suspen'siones resultan siempre inferiores a las reales, no pudiéndose seguir además, la evolución de estos últimos con el tiempo.

A pesar de ello, es evidente que las cinéticas de corrosión más elevadas y el ataque mayor lo presentan los cementos que inducen $\mathrm{pH}$ superiores (excepto los cementos con escorias de alto horno que contienen sulfuros), y que coinciden con un contenido en álcalis totales más elevardo.

La presentación de evidencia experimental de la existencia de un pH crítico o umbral a partir del cual el galvanizado no se pasiva, es sin duda un paso de capital importancia en favor de la resolución de la controversia mantenida actualmente en torno a la estabilidad de las armaduras galvanizadas en contacto con hormigón.

Así, cementos cuyo contenido en componentes menores $\left(\mathrm{Na}^{+}, \mathrm{K}^{+}\right.$y $\mathrm{SO}_{4}=$ principalmente), den lugar a un $\mathrm{pH}$ de la fase líquida que rellena los poros del hormigón, menor o igual que este pH umbral, pasivarán al galvanizado, mientras que hormigones o morteros que presenten un $\mathrm{pH}$ superior, desencadenaran cinéticas de disolución tanto más intensas cuanto mayor sea dicho $\mathrm{pH}$. 
En este contexto es importante recordar que, en general, los cementos norteamericanos presentan un contenido en álcalis pequeño debido al grave problema de áridos reactivos existentes en algunas regiones de USA, lo que explicaría los favorables resultados obtenidos por este país en el uso de armaduras galvanizadas.

\section{CONCLUSIONES}

- $\mathrm{El} \mathrm{pH}$ de una disolución saturada de $\mathrm{Ca}(\mathrm{OH})_{2}$ varía con la adición de $\mathrm{NaOH}, \mathrm{KOH}$ y $\mathrm{SO}_{4}{ }^{2}$ y el de una suspensión de cemento aumenta con el contenido en álcalis totales del mismo.

- Existe un pH umbral que marca una frontera entre la pasivación y la corrosión del galvanizado en contacto con un electrólito que simula la fase líquida del hormigón.

- La corrosión de las armaduras embebidas en mortero aumenta con el aumento del $\mathrm{pH}$ del filtrado de las suspensiones de los cementos.

- La Resistencia de Polarización ha resultado ser, una vez más, una técnica útil, rápida y relativamente exacta, que ha permitido seguir la evolución de la velocidad de corrosión.

\section{AGRADECIMIENTO}

Los autores agradecen a D. Francisco Huete la colaboración prestada en la realización de los ensayos y al Dr. J. A. González del CENIM sus interesantes sugerencias.

\section{B I B L I O G R A F I A}

(1) F. C. Porter: Concrete 8 (1976) 29.

(2) A. R. Cook and S. F. RADTKE: Intergalv 76 (11. Int. Galv. Conf.) Madrid Oct. (1976).

(3) K. W. J. Treadaway, B. L. Brown and R. N. Cox: ASTM-STP-713 (1980) 102.

(4) J. A. Gonzalez, A. J. Vazquez and C. Andrade: Matériaux et Construction (Rilem) (in press).

(5) C. Andrade and J. A. Gonzalez: Materiales de Construcción 172 (1978) 71.

(6) J. A. Gonzalez y C. Andrade: Rev. Iberoamericana de Corrosión y Protección 1 (1980) 15.

(7) L. LoNguet: Silicates Industriels $7 / 8$ (1976) 321.

(8) P. Longuet, L. Burglen and A. Zelwer: Rev. Matér. Const. et Travaux publics 676 (1973) 35.

(9) P. Delmas: Ciments, Bétons, Plâtres, Chaux 3 (1979) 167.

(10) C. Andrade and J. A. Gonzalez: Wertestoffe und Korrosion 29 (1978) 515.

(11) B. Roetheli, G. Cox and W. Littreal: Metals and Alloys 3 (1932) 73.

(12) W. Lieber and J. Gebauer: Zement-Kalk-Gips 4 (1969) 161.

(13) G. REHM and A. LAMMKK: Betonstein-Zeitung 36, 6 (1970) 360. 